Editorial

\title{
La uretrografía como primer paso para hacer una buena reconstrucción uretral
}

\author{
Jaime Andres Robayo ${ }^{1}$ Herney Andrés García-Perdomo ${ }^{10}$ \\ ${ }^{1}$ Departamento de Cirugia/Urología. Escuela de Medicina. \\ Universidad del Valle. Cali, Colombia
}

Urol Colomb 2021;30:1-2.

La uretrografía es la aplicación de medio de contraste a través de la uretra, con el objetivo de "pintarla" completamente y poder ver toda su anatomía. De esta manera, se logra definir si la uretra esta sana o por el contrario, tiene áreas enfermas. ${ }^{1}$ Hasta aquí, se podría considerar que es un estudio realmente sencillo y técnicamente fácil de realizar. Sin embargo, al llevarlo a la práctica, se trata de un estudio lleno de múltiples detalles que, si se tienen en cuenta, permitirán un mejor apoyo en la toma de decisiones en cuanto al diagnóstico y manejo de la estrechez uretral.

En medline (a través de Pubmed), la gran base de datos norteamericana, la palabra "urethrography" resulta en un poco mas de 900 articulos. La gran mayoría de los artículos habla sobre la técnica, interpretación, cómo mejorar los resultados y cómo disminuir la molestia del paciente. Llama la atención, que siendo un examen sencillo y con tanto soporte en la literatura, en la práctica clinica diaria, no se realiza con toda la técnica y todos los detalles recomendados. Normalmente el enfoque se hace en las áreas críticas y evidentes de estrechéz, pero la fase miccional, que es indispensable, por lo general no se hace y la maniobra de elongación uretral que es fundamental tampoco. ${ }^{2}$ Es, en muchas ocasiones, un examen traumático que se hace en ambientes hostiles donde se altera la tranquilidad del paciente e impide la fase miccional espontanea, en lugar de ofrecer un espacio tranquilo para poder realizarse con mayor comodidad y así obtener los mejores resultados. ${ }^{3}$ De otro lado, comunmente, los urólogos hemos delegado la realización de este examen tan indispensable en nuestra práctica al radiologo, quien logra realizar el examen, pero no logra dimensionar los detalles que el urólogo quiere ver. ${ }^{4}$

Dado lo expresado anteriormente, podríamos educar a varias de las generaciones de urólogos acerca de la adecuada técnica para realizar tan importante evaluación. Esta es una invitación a todos nuestros colegas, para que sea el urólogo, en

Address for correspondence Herney Andrés García-Perdomo, MD MSc, EdD, PhD, FACS,

Departamento de

Cirugía/Urología, Escuela de

Medicina, Universidad del Valle, Cali, Colombia

(e-mail: editorrevista@scu.org.co). tiempo real, quien realice el examen, vea los detalles y logre hacer una interpretación de la distensibilidad uretral, de las zonas de espongiofibrosis, de la apertura del esfínter externo, de la anatomía de la vejiga, su capacidad, su configuración, la apertura del cuello vesical, las diferentes características de la estrechez en la fase retrograda y anterógrada, y las medidas de las zonas criticas de estrechez, entre otros elementos fundamentales para un buen estudio diagnóstico. Tambien se pueden ofrecer algunos tips que permitan la micción del paciente como son: el uso de alfa bloqueador la noche anterior al estudio para facilitar la apertura del cuello vesical, realizarlo en ambientes cálidos, oscuros y silenciosos con la menor cantidad de personas presentes en la sala de procedimiento, estímulos para que el paciente orine como mojar manos o pies, percusión abdominal, escuchar chorro de agua, entre otros. Adicionalmente, en los últimos años el avance de endoscopios flexibles nos ha permitido incrementar el rendimiento diagnóstico de la prueba dada la introducción del mismo por vía suprapúbica y poder avanzar mas allá del cuello vesical en casos complejos; principalmente en pacientes que no logran hacer fase miccional. ${ }^{5}$

Parte del proceso de aprendizaje en reconstrucción uretral es predecir lo que se encontrará en el momento de llevar el paciente a cirugía y para esto es la uretrografía. La uretrografía nos indicará si es candidato a dilatación, a uretrotomia interna endoscópica o si por el contrario requiere una reconstrucción uretral. Tener claro el abordaje, las técnicas, la indicación para usar injertos y colgajos, y que esperar con la continencia de orina, hace que los resultados mejoren de forma signficativa y permite advertir al paciente sobre lo que esperamos al final del proceso de reconstrucción. Es por esto que, volviendo a nuestro título, la uretrografia debe ser el primer paso para una buena reconstrucción uretral.

Lo que nos depara el futuro con respecto al diagnostico de estrechez uretral esta basado en la ecografía, ${ }^{5}$ la resonancia
(C) 2021. Sociedad Colombiana de Urología. All rights reserved. This is an open access article published by Thieme under the terms of the Creative Commons Attribution-NonDerivative-NonCommercial-License, permitting copying and reproduction so long as the original work is given appropriate credit. Contents may not be used for commercial purposes, or adapted, remixed, transformed or built upon. (https://creativecommons.org/ licenses/by-nc-nd/4.0/)

Thieme Revinter Publicações Ltda., Rua do Matoso 170, Rio de Janeiro, RJ, CEP 20270-135, Brazil 
magnética ${ }^{6}$ y los modelos de reconstrucción tridimensional enfocados en la uretra. Este tipo de tecnología podría ser útil en casos complejos y muy particulares, sin embargo, por el momento el estándar para el diagnostico de la estrechez uretral sigue siendo la uretrografia. Un examen de facil acceso, con una técnica estándar, de bajo costo y que ofrece detalles fundamentales para el planeamiento de una intervención en aquellos pacientes que sufren de una patología uretral. ${ }^{6,7}$

\section{Referencias}

1 Flanagan JC, Batz R, Nordeck SM, Lemack GE, Brewington C. Urethrography for assessment of the adult male urethra [Internet]. Vol. 38, Radiographics Radiological Society of North America Inc.; 2018 [cited 2021 Feb 12]. p. 831-2. Available from: https://pubmed.ncbi.nlm.nih.gov/29757722/

2 Kathpalia R, Dalela D, Goel A, et al. Effect of phallic stretch on length of bulbous urethral stricture during retrograde urethrography. Urol Int 2014;93(01):63-66https://www. karger.com/Article/FullText/353228 cited2021Feb12 [Internet]
3 Angermeier KW, Rourke KF, Dubey D, Forsyth RJ, Gonzalez CM. SIU/ICUD consultation on urethral strictures: Evaluation and follow-up. Urology 2014;83(3, Suppl)S8-S17

4 Maciejewski C, Rourke K. Imaging of urethral stricture disease [Internet]. Vol. 4, Translational Andrology and Urology AME Publishing Company; 2015 [cited 2021 Feb 12]. p. 2-9. Available from: /pmc/articles/PMC4708283/

5 Li X, Sa YL, Xu YM, Fu Q, Zhang J. Flexible cystoscope for evaluating pelvic fracture urethral distraction defects. Urol Int 2012;89(04): 402-407https://www.karger.com/Article/FullText/339926 cited2021Feb12 [Internet]

6 Buckley JC, Wu AK, McAninch JW. Impact of urethral ultrasonography on decision-making in anterior urethroplasty. BJU Int 2012;109(03):438-442https://pubmed.ncbi.nlm.nih.gov/ 21615851/ cited2021Feb12 [Internet]

7 Osman Y, El-Ghar MA, Mansour O, Refaie H, El-Diasty T. Magnetic resonance urethrography in comparison to retrograde urethrography in diagnosis of male urethral strictures: is it clinically relevant? Eur Urol 2006;50(03):587-593, discussion 594https://pubmed.ncbi.nlm.nih.gov/16457942/ cited2021Feb12 [Internet] 